\title{
Manejo del dolor crónico en Atención Primaria, perfil de prescripción de opiáceos mayores: indicación, coste y efectos secundarios
}

\author{
M. Guzmán-Ruiz ${ }^{1}$, R. Mora-Moscoso², C.M. Delgado-Mediano ${ }^{1}$, A. Pérez-Milena ${ }^{1}$, M. Rueda-Rojas ${ }^{1}$ \\ y L.A. Gea-Rodríguez ${ }^{1}$ \\ ${ }^{1}$ Atención Primaria. Centro de Salud el Valle. Jaén. ${ }^{2}$ Servicio de Anestesiología, Reanimación y Terapia \\ del Dolor. Complejo Hospitalario Ciudad de Jaén. Jaén
}

Guzmán-Ruiz M, Mora-Moscoso R, Delgado-Mediano CM, Pérez-Milena A, Rueda-Rojas M, Gea-Rodríguez LA. Manejo del dolor crónico en Atención Primaria, perfil de prescripción de opiáceos mayores: indicación, coste y efectos secundarios. Rev Soc Esp Dolor 2014; 21(4): 197-204.

\begin{abstract}
Objectives: The main objective of the study was to determine the characteristics of major opioids prescribing in Primary Care.

Material and methods: A retrospective study was designed through the use of a historical cohort, assessing patients who have consumed major opiates in all Jaén city health centers during 2011, by systematic sampling, 215 patients, computing the sample size for a confidence level of $95 \%$, accuracy 5 $\%, 5 \%$ losses (expanding the $5 \%$ sample by reporting bias). Medical records were audited to collect the following data: Age, sex, medical history, social status, number of appointments and use of hospital resources, characteristics of opioids prescription (dose, duration, cost, adverse reaction) and use of other analgesics/adjuvants. Data were processed for a descriptive study and a subsequent comparison according to the prescribed opioid.

Results: 215 patients were included (44\% of total population) those obtained 323 prescriptions for opiates in the study year (23\% of the sample with two prescriptions of opiates and $13 \%$ with three). Most prescribed opioids were fentanyl (60\%) and buprenorphine (22\%) and the least prescribed is by $3 \%$ morphine. Buprenorphine is the most used in monotherapy (70\%), preferably in older women and pensioners, especially for joint pain by the general practitioner. The fentanyl is mainly used in patches (95\% of prescriptions of fentanyl) and hydromorphone is used in younger patients ( $<60$ years), prescribed
\end{abstract}

Recibido: 02-06-13.

Aceptado: 12-09-13. by the Pain and Rheumatology Departments (43\% and $20 \%$ of total prescriptions, respectively). Oxycodone is used in patients from areas with social transformation needs ( $46 \%$ of prescribed opioids in ZNTS) and exclusively for neuropathic and mixed pain $(52 \%$ and $48 \%$ respectively of total prescriptions of oxycodone), especially by the Rheumatology and Pain Unit. Morphine is used orally in cancer patients (46\% of prescriptions for morphine), half in retard formulation (45\% of prescriptions for morphine), and these patients were who have a higher healthcare demands (91\% of totals patients prescribed morphine, go to the emergency department, and $46 \%$ required hospital admission).

Conclusions: As improvement measure, after contrasting the results, we should encourage the prescription of morphine as an analgesic of choice in both acute and chronic severe pain. An improvement in the measuring instruments specific to each type of pain considering its pathogenesis is required, as well as fostering a greater use of rating scales, both at the start of treatment and during the follow-up of each patient to assess the evolution of pain and treatment effectiveness.

Key words: Primary Care. Analgesic opioid. Strong opioid. latrogenic disease. Drug costs.

\section{RESUMEN}

Objetivos: el objetivo principal del estudio es conocer las características de la prescripción de opiáceos mayores en Atención Primaria.

Material y métodos: se diseña un estudio retrospectivo mediante una cohorte histórica, valorando a los pacientes que hayan consumido opiáceos mayores en todos los centros de salud de Jaén capital durante 2011, mediante un muestreo sistemático, 215 pacientes, calculando el tamaño de muestra para un nivel de confianza $95 \%$, precisión $5 \%$, y pérdidas 5 $\%$ (ampliando la muestra este $5 \%$ por sesgo de información); Se auditan las historias clínicas recogiendo: edad, sexo, antecedentes personales, nivel social, frecuentación sanitaria, características de la prescripción de opiáceos (dosis, duración, coste, 
efectos secundarios) y uso de otros analgésicos/coadyuvantes. Se procesan los datos para ofrecer un estudio descriptivo y una posterior comparación en función del opiáceo prescrito.

Resultados: se valoran 215 pacientes (44\% del total de la población), de los que se obtienen 323 prescripciones de opiáceos en el año de estudio (23\% de la muestra con dos prescripciones de opiáceos y $13 \%$ con tres). Los opiáceos más prescritos son fentanilo (60\%) y buprenorfina (22\%), por un $3 \%$ de morfina. La buprenorfina es el más usado en monoterapia (70\%), preferentemente en mujeres de edad avanzada y pensionistas, sobre todo para el dolor mixto por parte del médico de familia. El fentanilo se usa principalmente en parches (95\% de las prescripciones de fentanilo) y la hidromorfona se emplea en pacientes de menor edad ( $<60$ años), prescrito por la Unidad del Dolor y Reumatología (43\% y 20 \% del total de prescripciones, respectivamente). La oxicodona se emplea más en pacientes de zonas necesitadas de transformación social (46\% del total de opioides prescritos en ZNTS) y exclusivamente para el dolor neuropático y mixto (52\% y $48 \%$ respectivamente de las prescripciones totales de oxicodona), sobre todo por parte de Reumatología y Unidad del Dolor (25 \% y $43 \%$ del total de prescripciones respectivamente). La morfina se emplea en pacientes oncológicos por vía oral (46\% de las prescripciones de morfina), la mitad en formulación retard ( $45 \%$ de las prescripciones de morfina), siendo estos pacientes quienes presentan una demanda sanitaria superior (91\% del total de pacientes con prescripción de morfina, acuden al servicio de urgencias, y un $46 \%$ precisan ingreso hospitalario).

Conclusiones: como medida de mejora, contrastando los resultados obtenidos, se debería fomentar la prescripción de morfina como analgésico de elección tanto en dolor agudo como en dolor crónico severo. Es preciso mejorar los instrumentos de medida específicos para cada tipo de dolor teniendo en cuenta la etiopatogenia de este, así como fomentar una mayor utilización de escalas de valoración, tanto al inicio del tratamiento, como durante el seguimiento de cada paciente para evaluar la evolución del dolor y la efectividad del tratamiento.

Palabras clave: Atención Primaria. Analgésicos opioides. Opiáceos mayores. Iatrogenia. Coste de medicamentos.

\section{INTRODUCCIÓN}

El dolor crónico es un importante problema de salud por su alta frecuencia, por las repercusiones que implica en el paciente y sus familiares, y por los altos costes económicos. En la atención sanitaria a un paciente con dolor crónico es preciso valorar la causa y la intensidad, datos necesarios para establecer un plan de tratamiento donde el médico tiene ante sí una doble tarea: educar al paciente y fijar metas realistas (1-3). La OMS propone el uso de la escalera analgésica, de forma pautada y realizando siempre una titulación de la necesidad de analgésicos así como de los tratamientos coadyuvantes que puedan ser necesarios $(1,4,5)$. El grupo farmacológico de los opioides está situado en el tercer escalón; su uso tiene un nivel de evidencia de tipo I-II, con un grado de recomendación B para su prescripción en el tratamiento del dolor crónico oncológico (6-8) y no oncológico $(6,9,10)$, aunque se precisan más estudios sobre los efectos en tratamientos a largo plazo (8,10-13). Las evidencias son algo inferiores (II-III) para el tratamiento del dolor neuropático, precisándose en estos pacientes el empleo de mayores dosis en comparación con los pacientes con dolor nociceptivo $(6,14,15)$. Dentro del uso de los analgésicos opiáceos, la prescripción de la morfina permite obtener una mejor efectividad analgésica y un incremento en la seguridad de los pacientes (16). El uso del resto de los opioides mayores debería efectuarse para casos de intolerancia a la morfina, a la ingesta oral, en rotación de opioides, o en determinadas indicaciones concretas $(15,16)$.

El aumento de la prevalencia de las enfermedades crónicas en la población atendida genera una mayor prevalencia de pacientes con dolor crónico que parece estar infraevaluado e infratratado (4). En este contexto, el uso de opioides en Atención Primaria constituye una excelente opción para el tratamiento del dolor crónico. Históricamente su prescripción ha sido deficiente debido a la ausencia de medidas objetivas o fisiológicas del dolor, la falta de experiencia en el tratamiento del dolor crónico o el temor a los efectos adversos y los trastornos coexistentes (como la adicción, la falta de interés en el manejo del dolor y la preocupación del médico acerca del uso indebido) $(2,6,17$ 20). En las últimas tres décadas, por el contrario, parece existir un cambio de actitud en Atención Primaria sobre el uso de los opioides con una actitud positiva por parte de los médicos que ha permitido incrementar la experiencia en la evaluación del dolor y mejorar el control del mismo $(18,21,22)$.

La heterogeneidad de las etiologías que causan dolor crónico y el amplio arsenal analgésico del que dispone el médico de familia conforman un panorama de actitudes terapéuticas muy diverso en Atención Primaria (23). La necesidad de conocer las características de la prescripción de opiáceos mayores en Atención Primaria se convierte, así, en el objetivo del presente estudio que también pretende valorar el perfil del paciente que consume opiáceos mayores y cuantificar los efectos secundarios y el coste económico del uso de esta medicación.

\section{MATERIAL Y MÉTODOS}

Se diseña un estudio transversal descriptivo incluyendo todos los centros de salud de una capital urbana andaluza (Jaén), que atienden una población total de 116.781 habitantes de los cuales un $20 \%$ viven en zonas necesitadas de transformación social (ZNTS). La población diana está compuesta por toda la población mayor de 14 años que haya consumido opiáceos mayores durante el año 2011, por cualquier motivo e independientemente del principio 
activo empleado, la duración o la dosis del tratamiento. Se eligen 215 pacientes de entre todos aquellos que han tomado opiáceos mayores durante ese periodo de tiempo (lo que supone un $44 \%$ del total), mediante un muestreo sistemático del listado obtenido en el servicio de farmacia del Distrito Sanitario, calculándose en base a una prevalencia de uso de opiáceos en el $17 \%$ de la población, con un nivel de confianza del $95 \%$ y una precisión del $5 \%$. Se aumenta la muestra un $5 \%$ más para evitar las pérdidas por historias clínicas mal cumplimentadas.

La fuente de información empleada es la historia clínica digital (Diraya), obteniendo los valores de las siguientes variables de cada paciente:

- Variables sociodemográficas: edad (en años), sexo, ocupación (activo/pensionista), pertenencia o no a una ZNTS.

- Variables clínicas: antecedentes personales de enfermedad, frecuentación sanitaria durante el año 2011 (número de consultas realizadas a la consulta de medicina y enfermería de familia, número de avisos domiciliarios, número de consultas a urgencias e ingresos hospitalarios).

- Prescripción de opiáceos mayores: principio activo, dosis, duración del tratamiento (en meses), vía de administración, precio/envase y ${ }^{\circ}{ }^{\circ}$ de envases retirados de la farmacia durante el año 2011; efectos secundarios e interacciones; médico prescriptor; uso de escalas de valoración del dolor.

- Motivo de la prescripción: indicación del tratamiento según el tipo de dolor, clasificando a los sujetos según exploración clínica y resultado de escalas de valoración de dolor (LANSS, DN4) en dolor: neuropático, nociceptivo, mixto e irruptivo $(24,25)$ (Tabla I). La clasificación de los pacientes en cada uno de los cuatro grupos se realizó según las características clínicas del dolor que presentaban en la exploración física, así como el resultado de los test de valoración del tipo de dolor que estaban registradas en las historias clínicas; posteriormente realizamos la tabla I con el diagnóstico principal de cada paciente.

- Otras variables farmacológicas: uso de otros fármacos analgésicos/coadyuvantes (AINE, analgésicos no opioides, antidepresivos, antiepilépticos); número de fármacos activos durante el año 2011.

Para el análisis estadístico se ha empleado el software SPSS (v21). Se comprobó la normalidad de los datos mediante el Test de Shapiro-Wilk. Se realizó inicialmente un análisis descriptivo de las principales variables: para las variables cualitativas se obtuvo su distribución de frecuencias (número de casos y porcentaje) y para las variables cuantitativas la media con su correspondiente desviación típica. Posteriormente se llevó a cabo un análisis bivariante comparando las características de las variables estudiadas en cada grupo de pacientes con uso de diferentes opiáceos mayores, comparando las variables cualitativas mediante la prueba de la $\chi^{2}$ (o test de Fisher) y las cuantitativas con el análisis de la varianza (ANOVA) (o prueba de KruskallWallis si no siguen una distribución normal). Se fijó un nivel de significación de $\mathrm{p}<0,05$.

\section{RESULTADOS}

\section{Tipos y dosis de opiáceos empleados}

De los 215 pacientes seleccionados se recogen 323 prescripciones de opiáceos mayores, ocupando el fentanilo y la buprenorfina más del $80 \%$ de las prescripciones (Tabla II). La media de opiáceos empleados en cada paciente es de 1,8 $\pm 0,8$, siendo superior en el caso de la morfina $(2,2 \pm 0,8)$ y

TABLA I. AGRUPACIÓN DE LAS PATOLOGÍAS EN LOS PACIENTES DEL ESTUDIO SEGÚN LA ETIOLOGÍA Y CLÍNICA DEL MISMO

\begin{tabular}{llll}
\hline \multicolumn{1}{c}{ Nociceptivo } & \multicolumn{1}{c}{ Mixto } & \multicolumn{1}{c}{ Irruptivo } & \multicolumn{1}{c}{ Neuropático } \\
\hline Fracturas vertebrales & Artropatía crónica & Ca. cérvix & Neuropatía diabética \\
Fracturas de cadera & - Coxartrosis & Ca. vejiga & Plexopatía braquial cervical \\
Escoliosis & - Discartrosis & Leucemia & Vertebroplastia lumbar \\
Hipercifosis & - Espondiloartrosis & Ca. esófago & Necrosis aséptica femoral \\
Esclerosis subcondral & - Cervicoartrosis & Ca. mama & Microdisectomía lumbar \\
Condromatosis & Poliartralgias & Mieloma & Parálisis braquial \\
Estenosis canal medular & Fibromialgia & Ca. colon & Amputación extremidades \\
Trocanteritis & Artropatía hiperuricémica & Ca. próstata & Laminectomía vertebral \\
Epicondilitis & Artritis reumatoide & Ca. pulmón & Mastectomía dcha. \\
Bursitis acromiodeltoidea & Polimialgia & & Fibrosis lumbar \\
& Espondilitis anquilosante & & Sida \\
& Espondililisis cérvico-dorsal & & Hernia discal \\
& Osteoporosis & & \\
\hline
\end{tabular}


Rev. Soc. Esp. del Dolor, Vol. 21, N. ${ }^{\circ}$ 4, Julio-Agosto 2014

la oxicodona $(2,4 \pm 0,7)(\mathrm{p}<0,001$ ANOVA). Un $74 \%$ de los pacientes consumen un solo opiáceo, un $23 \%$ toman dos opiáceos y un $13 \%$ consumen tres opiáceos. Un $24 \%$ de los pacientes consumen dos opiáceos iguales con diferente presentación farmacológica y/o dosis, y en un $9 \%$ hasta tres opiáceos iguales. El fármaco más empleado en monoterapia es la buprenorfina en un $70 \%$, mientras que la oxicodona y la morfina no superan el $20 \%$ como tratamiento único. Por el contrario, la oxicodona se asocia a dos opiáceos en más de la mitad de los casos donde es utilizado (52\%).

Ha existido menos de un $1 \%$ de interacciones farmacológicas, todas en las prescripciones de fentanilo; también se han registrado un $4 \%$ de efectos secundarios, más frecuente en los consumidores de oxicodona (Tabla II). Se han empleado otros fármacos para el dolor, siendo los más utilizados los analgésicos no opioides (paracetamol, metamizol) en un $82 \%$ de los casos, seguidos de los ansiolíticos (67\%), AINE (59\%), antidepresivos (56\%), tramadol (36\%) y antiepilépticos (29\%), con similar distribución en todos los grupos.

\section{Características de los sujetos}

La edad media de los pacientes incluidos en el estudio es de 71,2 años $( \pm 13,3)$, siendo superior en los pacientes consumidores de buprenorfina, fentanilo y oxicodona frente a los que toman hidromorfona y morfina, que es inferior a los 60 años de media ( $\mathrm{p}<0,001$ test ANOVA) (Tabla II). La mayoría de los sujetos estudiados son mujeres (78\%), sobre todo cuando se prescribe buprenorfina, fentanilo e hidromorfona, con una diferencia cercana al $20 \%$ con oxicodona y morfina $\left(\mathrm{p}<0,05\right.$ test $\left.\chi^{2}\right)$. Los pacientes tienen una media de $6( \pm 2,7)$ patologías crónicas, siendo las más frecuentes las enfermedades del aparato locomotor $(90 \%)$, las enfermedades cardiovasculares (46\%) y la HTA (44\%). Un $19 \%$ de los sujetos estudiados pertenecen a una zona necesitada de transformación social (ZNTS), con un mayor consumo de oxicodona ( $46 \%$ ) que presenta diferencias significativas con respecto al resto: hidromorfona (20\%), fentanilo (19\%), y en menos de un $10 \%$ buprenorfina y morfina $\left(\mathrm{p}<0,001 \chi^{2}\right)$.

Los pacientes acuden de media al año 10 veces $( \pm 0,7)$ al médico de familia y 6,9 veces $( \pm 8,8)$ a la enfermería de su Centro de Salud ( $\mathrm{p}<0,01$ ANOVA). La media de consultas al servicio de urgencias hospitalarias es de 1,5 veces $( \pm$ $1,8)$. El $19 \%$ de los pacientes han requerido avisos domiciliarios por parte de su médico de familia, destacando los consumidores de fentanilo y los de buprenorfina. Los pacientes consumidores de morfina destacan, con diferencias estadísticamente significativas, como los más frecuentadores de enfermería y de urgencias, precisando un mayor número de ingresos hospitalarios, seguidos de los tratados con fentanilo y buprenorfina (Tabla II).
Se han empleado escalas de valoración del dolor (EVA) en sólo un $7 \%$ de los pacientes, con diferencias significativas según el tipo de opiáceo: su uso es más frecuente en los consumidores de hidromorfona y oxicodona ( $\mathrm{p}<0,001$ test $\chi^{2}$ ); en todos los casos se trataban de pacientes tratados en la Unidad del Dolor.

\section{Tipos de dolor}

La distribución del consumo de opiáceos mayores según el tipo de dolor se muestra en la figura 1. Predomina el dolor de origen mixto y neuropático. La morfina se emplea en casi la mitad de los casos en pacientes oncológicos, mientras que la oxicodona se prescribe exclusivamente para dolor neuropático y mixto. La hidromorfona tiene mayor uso que el resto en dolor nociceptivo, y el fentanilo y la buprenorfina se emplean sobre todo en dolor mixto ( $p$ $<0,05 \chi^{2}$ ).

Las dosis empleadas de cada fármaco varían en función de la etiología del dolor, como puede apreciarse en la tabla II. Un $38 \%$ de los pacientes precisan aumentos de dosis del opiáceo inicialmente prescrito, sin existir diferencias significativas en las necesidades de incremento de dosis para cada tipo de opiáceos empleado. El fentanilo y la buprenorfina se emplean en su mayoría por vía tópica, usando como medio de administración el parche, mientras que el resto se utilizan por vía oral $\left(\mathrm{p}<0,001 \chi^{2}\right)$ (Tabla II).

Existen diferencias estadísticamente significativas en cuanto a la prescripción de opiáceos según especialidad, siendo el médico de familia el principal prescriptor. Oncología prescribe principalmente morfina, Traumatología prescribe por vía tópica, mientras que el resto de especialistas hospitalarios prescriben oxicodona e hidromorfona en diferentes porcentajes ( $\left.<<0,001 \chi^{2}\right)$ (Fig. 2).

\section{Coste del tratamiento}

El coste medio del tratamiento con opiáceos mayores por paciente y año es de $884,64 €( \pm 1741,45)$. El precio aumenta cuando se indican dos fármacos opiáceos, pero sobre todo si hay tres opioides prescritos a la vez ( $\mathrm{p}<0,01$ ANOVA de 1 vía), que duplica los costes anteriores. Así, el coste medio del tratamiento con un solo opiáceo es de $716,56 €( \pm 1280,79)$, con dos opiáceos de 854,70 € ( \pm $1.413,23)$ y con tres opiáceos alcanza los $1.724,47 €( \pm$ $3.302,20)$. No hay diferencias significativas para el sexo, la edad o el tipo de dolor en el coste medio del tratamiento con opiáceos por paciente y año. Para el coste por tipo de opiáceo no existen diferencias estadísticamente significativas pero el coste de la buprenorfina y del fentanilo es de dos a tres veces superior que el del resto de opiáceos (Tabla III). 
TABLA II. CARACTERÍSTICAS DE LAS VARIABLES ESTUDIADAS SEGÚN EL TIPO DE OPIÁCEO MAYOR CONSUMIDO

\begin{tabular}{|c|c|c|c|c|c|}
\hline & Buprenorfina & Fentanilo & Oxicodona & Hidromorfona & Morfina \\
\hline Prevalencia & $22 \%$ & $60 \%$ & $9 \%$ & $6 \%$ & $3 \%$ \\
\hline Pensionista (*) & $88 \%$ & $94 \%$ & $100 \%$ & $45 \%$ & $64 \%$ \\
\hline Edad media de uso (¥) & $72,5 \pm 10,7$ & $72,6 \pm 13,0$ & $74,0 \pm 12,8$ & $58,5 \pm 13,6$ & $59,1 \pm 16,7$ \\
\hline N. ${ }^{o}$ A. Personales: & $6,2 \pm 2,3$ & $6,1 \pm 2,6$ & $7,3 \pm 3,3$ & $6,3 \pm 3,6$ & $4,4 \pm 2,2$ \\
\hline DM & $28 \%$ & $26 \%$ & $45 \%$ & $25 \%$ & $18 \%$ \\
\hline $\operatorname{HTA}(+)$ & $36 \%$ & $46 \%$ & $69 \%$ & $30 \%$ & $18 \%$ \\
\hline $\mathrm{CV}(+)$ & $51 \%$ & $44 \%$ & $55 \%$ & $40 \%$ & -- \\
\hline Neumología (+) & $6 \%$ & $20 \%$ & $28 \%$ & $10 \%$ & $9 \%$ \\
\hline Locomotor & $93 \%$ & $89 \%$ & $93 \%$ & $100 \%$ & $73 \%$ \\
\hline Salud mental (+) & $41 \%$ & $20 \%$ & $14 \%$ & $20 \%$ & $27 \%$ \\
\hline \multicolumn{6}{|l|}{ Frecuentación: } \\
\hline MAP & $10,6 \pm 14,0$ & $9,3 \pm 7,4$ & $9,3 \pm 8,4$ & $12,1 \pm 8,1$ & $11,1 \pm 5,6$ \\
\hline Enfermería (+) & $7,3 \pm 9,6$ & $7,4 \pm 8,4$ & $7,4 \pm 7,2$ & $2,4 \pm 3,3$ & $17,9 \pm 28$ \\
\hline Urgencias & $65 \%$ & $59 \%$ & $59 \%$ & $60 \%$ & $91 \%$ \\
\hline Ingreso hospitalario (\#) & $15 \%$ & $16 \%$ & -- & $10 \%$ & $46 \%$ \\
\hline Domicilio & $20 \%$ & $21 \%$ & $14 \%$ & $5 \%$ & -- \\
\hline Dosis $(\mu g-m g / d i a):$ & $19,6 \pm 25,6 \mu g$ & $53,4 \pm 177,7 \mu \mathrm{g}$ & $32,3 \pm 33,2 \mathrm{mg}$ & $9,0 \pm 4,3 \mathrm{mg}$ & $41,2 \pm 47 \mathrm{mg}$ \\
\hline Nociceptivo & $13,3 \pm 5,7$ & $177,4 \pm 500,1$ & -- & $14,0 \pm 2,8$ & $10,0(\mathrm{vu})$ \\
\hline Mixto & $18,6 \pm 11,1$ & $17,8 \pm 14,4$ & $35,2 \pm 46,1$ & $9,1 \pm 5,0$ & $20(\mathrm{vu})$ \\
\hline Irruptivo & $11,7 \pm 0,0$ & $77,3 \pm 216,7$ & -- & -- & $70,0 \pm 70,0$ \\
\hline Neuropático & $25,3 \pm 47,0$ & $77,0 \pm 162,1$ & $30,0 \pm 20,5$ & $7,4 \pm 2,7$ & $30,0 \pm 26,5$ \\
\hline \multicolumn{6}{|l|}{ Vía administración (*): } \\
\hline Oral & $1 \%$ & $3 \%$ & $100 \%$ & $100 \%$ & $100 \%$ \\
\hline Transdérmico & $99 \%$ & $95 \%$ & $0 \%$ & $0 \%$ & $0 \%$ \\
\hline Transmucosa & $0 \%$ & $2 \%$ & $0 \%$ & $0 \%$ & $0 \%$ \\
\hline \multicolumn{6}{|l|}{ Presentación (*): } \\
\hline Parche & $99 \%$ & $95 \%$ & -- & -- & $9 \%$ \\
\hline Oral & $1 \%$ & $1 \%$ & $21 \%$ & $20 \%$ & $46 \%$ \\
\hline Oral Retard & -- & -- & $79 \%$ & $80 \%$ & $45 \%$ \\
\hline Pulverizador nasal & -- & $1 \%$ & -- & -- & -- \\
\hline Intramucoso & & $3 \%$ & -- & -- & -- \\
\hline Aumento de dosis & $20 \%$ & $44 \%$ & $45 \%$ & $30 \%$ & $46 \%$ \\
\hline Efectos secundarios & $3 \%$ & $3 \%$ & $10 \%$ & $5 \%$ & $0 \%$ \\
\hline Uso de EVA (\$) & -- & $7 \%$ & $14 \%$ & $25 \%$ & $9 \%$ \\
\hline
\end{tabular}

DM: diabetes mellitus. HTA: hipertensión arterial. CV: enfermedad cardiovascular. MAP: médico de Atención Primaria. EVA: escala visual analógica. Diferencias significativas: $(*) p<0,001 \chi^{2}$; (¥) $\mathrm{p}<0,001$ ANOVA; $(+) \mathrm{p}<0,01$ ANOVA; (\#) $\mathrm{p}<0,05 \chi^{2} ;(\$) \mathrm{p}<0,001 \chi^{2}$.

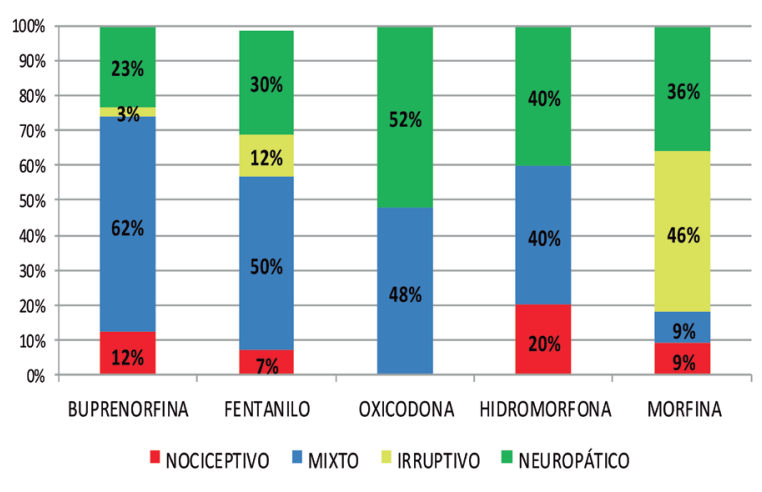

Diferencias significativas con $p<0,05$ test $x^{2}$

Fig. 1. Distribución del consumo de opiáceos mayores según el tipo de dolor.

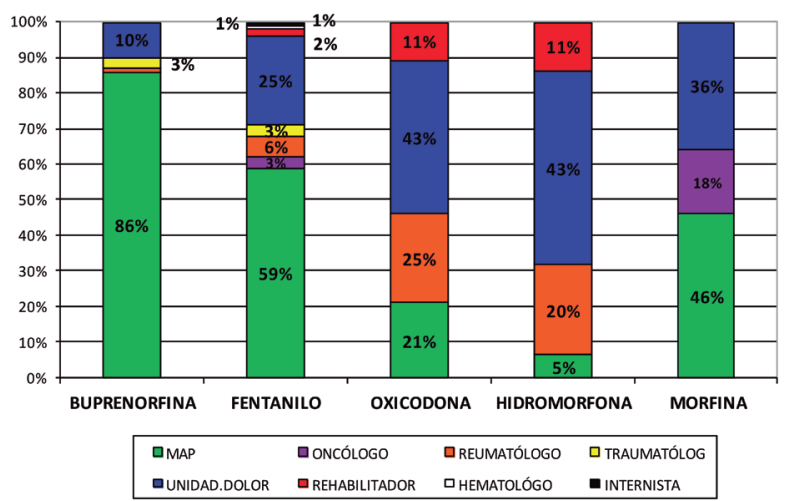

Fig. 2. Distribución de la prescripción de los diferentes opiáceos según el tipo de médico prescriptor. 
Rev. Soc. Esp. del Dolor, Vol. 21, N. ${ }^{\circ}$ 4, Julio-Agosto 2014

TABLA III. COSTE MEDIO DEL TRATAMIENTO CON OPIÁCEOS POR PERSONA Y AÑO EN FUNCIÓN DEL TIPO DE OPIÁCEO PRESCRITO

\begin{tabular}{lcccccc}
\hline & $n$ & Media & Desviación típica & Error típico & \multicolumn{2}{c}{ Intervalo de confianza al 95 \% } \\
\hline Buprenorfina & 57 & $1156,1 €$ & $1694,5 €$ & $224,4 €$ & $7706,5 €$ & $1605,7 €$ \\
Fentanilo & 126 & $897,3 €$ & $1933,9 €$ & $172,9 €$ & $556,3 €$ & $1238,3 €$ \\
Oxicodona & 11 & $399,3 €$ & $560,5 €$ & $168,9 €$ & $22,8 €$ & $775,7 €$ \\
Hidromorfona & 13 & $258,9 €$ & $252,8 €$ & $70,1 €$ & $106,2 €$ & $411,7 €$ \\
Morfina & 7 & $478,7 €$ & $727,4 €$ & $274,9 €$ & $194,0 €$ & $1151,5 €$ \\
Total & 215 & $884,6 €$ & $1741,4 €$ & $118,8 €$ & $650,5 €$ & $1118,7 €$ \\
\hline
\end{tabular}

Diferencias significativas entre los diferentes opiáceos con $\mathrm{p}<0,01$ test ANOVA.

\section{DISCUSIÓN}

Los resultados obtenidos ofrecen las principales características de la prescripción de opioides mayores en Atención Primaria. La elección del tipo de opiáceo mayor parece depender no sólo de la etiología del dolor sino también de la edad, el sexo y el nivel social del paciente (26), algo que condiciona las diferencias en la utilización de servicios sanitarios en cada grupo farmacológico. El médico prescriptor también desempeña un papel central en la elección del tratamiento que parece estar ligado a su formación específica y a sus experiencias previas en el campo de la analgesia $(3,20)$.

Por otra parte, el uso de los analgésicos opioides no queda relegado al tratamiento del dolor oncológico sino que se emplea para múltiples patologías relacionadas con diferentes especialidades. El fentanilo y la buprenorfina por vía transdérmica son los opiáceos más prescritos para los pacientes con dolor, como ocurre en el resto de Andalucía y de España (27), estando prescritos sobre todo para patología de tipo locomotor $(22,28,29)$. Estos dos opioides están disponibles en la formulación transdérmica y en base a los datos publicados ambos parecen ser eficaces, de baja toxicidad y buenos perfiles de tolerabilidad, especialmente a dosis bajas $(6,9)$. Este hecho puede estar relacionado con su alta prescripción sobre todo teniendo en cuenta que la preocupación fundamental acerca de la eficacia a largo plazo de los opiáceos es la seguridad y en particular el riesgo de tolerancia, dependencia o abuso $(11,12)$. Otros opioides empleados en menor porcentaje son la oxicodona, utilizada preferentemente para el dolor neuropático, y la hidromorfona, para el dolor nociceptivo.

La morfina pasa a ser un analgésico de elección para el dolor oncológico (en pacientes con un pronóstico de enfermedad terminal) (8) y prescrito de forma exclusiva por parte de oncólogos, médicos de familia y médicos de la unidad del dolor, quedando en general como un analgésico de segunda elección frente a los otros opioides. Se ha comprobado un decremento de su prescripción en los últimos años en España y Europa (28-30). Sin embargo, se considera el analgésico opiáceo de elección por su coste-eficacia en cualquier tipo de dolor severo agudo o crónico $(15,17)$ dado que ningún otro tipo de opiáceo mayor supera a la morfina en eficacia, flexibilidad de empleo, coste y seguridad (16). La escasa prescripción de la morfina, así como del resto de opiáceos mayores, puede estar muy relacionado con el desconocimiento de la farmacodinámica de este opiáceo y el temor a los efectos secundarios por parte de los médicos prescriptores $(3,17,19,28-30)$, el temor de los pacientes y sus familias motivados por creencias sociales o religiosas (5), y las medidas legislativas que incrementan la burocracia de su prescripción $(7,20,28)$. Tampoco es despreciable la presión que pueda ejercer la industria farmacéutica para promocionar opiáceos nuevos diferentes a la morfina (2830). Sin embargo, su dosificación y administración es fácil para el facultativo y el paciente $(17,21,27)$ y no parece provocar una excesiva iatrogenia tras una adecuada titulación (6).

El presente estudio muestra un pequeño porcentaje de efectos secundarios, hecho que podría deberse a un adecuado uso de esta medicación, aunque sería necesario averiguar si existe un sesgo de información (al no registrar los efectos secundarios en las historias clínicas) o se debe al uso de dosis bajas de los opioides. En la experiencia de los pacientes que usan opiáceos, la toma de esta medicación no produjo conductas adictivas, secundarismos graves o problemas de tolerancia a pesar de los temores iniciales $(5,13,23)$.

El uso de dos opiáceos conjuntos parece la norma habitual en la prescripción de este tipo de analgésicos, indicando uno de ellos como rescate para el dolor irruptivo. En este estudio, la cuarta parte de los pacientes que consumen dos opiáceos toman el mismo principio activo pero con diferente presentación farmacológica, pero a su vez encon- 
tramos un gran número de pacientes que presentaban varias prescripciones contemporáneas de igual principio activo, con idéntica presentación farmacológica, siendo ambas prescripciones de baja concentración del opioide, habiendo podido utilizarse una única prescripción del mismo fármaco, pero con una mayor concentración. Esto puede indicar una inadecuada prescripción de la medicación de rescate o bien una mala estrategia de aumento de dosis. Además, esta circunstancia encarece mucho el coste económico de la prescripción analgésica realizada. Como alternativa a la suma de prescripciones, se deberían utilizar correctamente los fármacos analgésicos de otros escalones así como otras terapias adyuvantes $(1,21)$. Tanto la adición de diferentes opiáceos como el uso de principios activos diferentes provoca un encarecimiento del tratamiento analgésico sin asegurar por ello una mayor eficacia.

Por otro lado, las dosis utilizadas para cada opiáceo mayor son variables y dependen fundamentalmente del tipo del dolor que origine la prescripción y de su intensidad. Más de la tercera parte de los pacientes estudiados necesitan un incremento de dosis para el control del dolor. Los consumidores de morfina son quienes presentan un mayor aumento de dosis, siendo pacientes en su mayoría con dolor de tipo oncológico con muchos episodios irruptivos. Estos datos son discrepantes con otros estudios donde se aprecia que la mayor dosis de medicación se emplea en los pacientes con dolor nociceptivo (6) o neuropático (14) y pueden deberse a un mayor seguimiento médico de los síntomas del paciente oncológico.

El sesgo de información es la principal limitación del estudio, dado que todos los datos se recogen de la historia clínica. Por tanto, se desconoce tanto el grado de adherencia al tratamiento analgésico como la satisfacción o la mejora del dolor que experimenta el paciente. Además de los posibles errores en el registro de la exploración física y características clínicas del tipo de dolor de cada paciente. También puede ocurrir que se trate un elevado número de pacientes, pero con dosis inferiores a las definidas por la OMS o durante menos tiempo del adecuado, algo que este estudio puede no detectar.

Hay, al mismo tiempo, un escaso registro de la valoración del dolor mediante escalas validadas, por mal registro o por ser una herramienta poco utilizada fuera de las Unidades del Dolor. Por tanto, se precisan el uso de instrumentos de medida específicos para cada tipo de dolor, para realizar un adecuado seguimiento y registro del dolor y poder valorar la efectividad del tratamiento, con una mayor adecuación a las guías de práctica clínica basadas en la evidencia que permitan una atención más coordinada y eficaz ante el dolor grave $(10,14,20,27,31)$.

Al margen de disparidades en el uso de los diferentes opiáceos hallados en este estudio, lo más importante es que la utilización de analgésicos potentes se va generalizando y que un elevado porcentaje de pacientes no oncológicos están siendo ya tratados con opioides para poder quitar o aliviar su dolor (28). Sin embargo existen escasas referencias sobre la eficacia del tratamiento opiáceo para el dolor crónico no oncológico en seguimientos superiores a 12 semanas, lo que debe hacer individualizar cada terapia analgésica en función de la respuesta al dolor (11-13,25).

Se precisa, además, establecer una comunicación eficaz entre los diferentes especialistas hospitalarios, y a su vez con los médicos de familia para homogeneizar las prescripciones analgésicas en función de la gravedad y el tipo de dolor (19), realizando protocolos multidisciplinares. No solamente deben ser tenidos en cuenta estos criterios, sino que el tratamiento con opioides debe valorar la eficacia global del opiáceo, los posibles efectos secundarios, el inicio de la acción, las interacciones farmacológicas o el potencial de abuso, así como otras cuestiones prácticas como el coste del fármaco, para establecer así un equilibrio entre la medicina basada en la evidencia y la experiencia personal de cada facultativo $(6,10,14,25)$.

Es preciso, además de la adecuada prescripción de analgésicos opioides, un enfoque integral del dolor que permita al paciente adoptar un papel activo en su enfermedad y mantener un adecuado estado funcional $(5,6,17,23)$.

\section{AGRADECIMIENTOS}

Queremos agradecer a María Dolores Morales (farmacéutica del Distrito Sanitario de Jaén, del Servicio Andaluz de Salud) su ayuda para la realización de este proyecto.

\author{
CORRESPONDENCIA: \\ Marta Guzmán-Ruiz \\ Atención Primaria \\ Centro de Salud el Valle \\ Rda. Alcalde García Segovia, s/n \\ 23009 Jaén \\ e-mail: martaguzru_85@hotmail.com
}

\section{BIBLIOGRAFÍA}

1. Ariz MJ, Meléndez A. Actualización en el manejo del dolor crónico. Uso racional de la escala analgésica de la OMS. Boletín de información farmacoterapéutica de Navarra; 2004;12(4).

2. Jackma RP, Purvis JM, Mallett BS. Chronic nonmalignant pain in Primary Care. Am Fam Physician 2008;15:1155-62.

3. Bope ET, Douglass AB, Gibovsky A, Jones T, Nasir L, Palmer T, et al. Pain management by the family physician: the family practice pain education project. J Am Board Fam Pract 2004;17:S1-12.

4. Guerra de Hoyos JA, Álvarez González J, et al. Consejería de Salud 2010. Plan Andaluz de Atención a las Personas con Dolor 2010-2013. 
5. Blake S, Ruel B, Seamark C, Seamark D. Experiences of patients requiring strong opioid drugs for chronic non-cancer pain: A patient-initiated study. Br J Gen Pract 2007;57:101-8.

6. Pergolizzi J, Böger RH, Budd K, Dahan A, Erdine S, Hans $\mathrm{G}$, et al. Opioids and the management of chronic severe pain in the elderly: Consensus statement of an International Expert Panel with focus on the six clinically most often used World Health Organization Step III opioids (buprenorphine, fentanyl, hydromorphone, methadone, morphine, oxycodone). Pain Pract 2008;8:287-313.

7. Wolfert MZ, Gilson A M, Dahl JL, Cleary JF. Opioid analgesics for pain control: Wisconsin physicians' knowledge, beliefs, attitudes, and prescribing practices. Pain Med 2010;11:425-34.

8. Colson J, Koyyalagunta D, Falco FJE, Manchikanti L. A Systematic review of observational studies on the effectiveness of opioid therapy for cancer pain. Pain Physician 2011;14:E85-E102.

9. Przeklasa-Muszy ska A, Dobrogowski J. Transdermal buprenorphine in the treatment of cancer and non-cancer pain - the results of multicenter studies in Poland. Pharmacol Rep 2011;63:935-48.

10. Kahan M, Mailis-Gagnon A, Wilson L, Srivastava A; National Opioid Use Guideline Group. Canadian guideline for safe and effective use of opioids for chronic noncancer pain: Clinical summary for family physicians. Can Fam Physician 2011;57:1257-76.

11. Manchikanti L, Ailinani H, Koyyalagunta D, Datta S, Singh V, Eriator I, Sehgal N, et al. A systematic review of randomized trials of long-term opioid management for chronic non-cancer pain. Pain Physician 2011;14:91-121.

12. Manchikanti L, Vallejo R, Manchikanti KN, Benyamin RM, Datta S, Christo PJ. Effectiveness of long-term opioid therapy for chronic non-cancer pain. Pain Physician 2011;14:E133-56.

13. Watson CP, Watt-Watson J, Chipman M. The long-term safety and efficacy of opioids: A survey of 84 selected patients with intractable chronic noncancer pain. Pain Res Manag 2010;15:213-7.

14. Finnerup NB, Otto M, Jensen TS, Sindrup SH. An evidence-based algorithm for the treatment of neuropathic pain. MedGenMed 2007;9:36.

15. Escuela Andaluza de Salud Pública - Servicio Andaluz de Salud - Consejería de Salud y bienestar social. Grado de utilización en atención primaria de opioides de tercer escalón por tipo. Andalucía, otras Comunidades Autónomas y España, 2008. Resultados y Calidad del Sistema Sanitario Público de Andalucía. Edición 2012.

16. Grupo Multidisciplinar Asesor en uso Racional del Medicamento Servicio Andaluz de Salud. Medicamentos para el dolor: selección de opioides. Áreas de mejora en el uso racional de medicamentos y criterios de calidad de pres- cripción. Informe justificativo de criterios de selección de medicamentos;2011:1-12.

17. Gooberman-Hill R, Heathcote C, Reid CM, Horwood J, Beswick AD, Williams S, et al. Professional experience guides opioid prescribing for chronic joint pain in primary care. Family Practice 2011; 28:102-9.

18. Caramés MA, Clavo B, Rodríguez-Salido MJ, García-Cortés J, Saavedra T. Opioides y atención primaria. Aspectos prácticos. Ponencia presentada en el "V Foro Atlántico del dolor" en Las Palmas de Gran Canaria; 2004.

19. Barry DT, Irwin KS, Jones ES, Becker WC, Tetrault JM, Sullivan LE, et al. Opioids, chronic pain, and addiction in primary care. J Pain 2010; 11:1442-50.

20. Galvez R. Variable use of opioid pharmacotherapy for chronic non cancer pain in Europe: Causes and consequences. J Pain Palliat Care Pharmacother 2009;23:346-56.

21. Sinatra R. Opioid analgesics in primary care: Challenges and new advances in the management of noncancer pain. Journal of the American Board Family Medicine 2006;2:165-77.

22. Canal Sotelo J, Barceló Montalà $\mathrm{A}$, Rosauro Gómez MJ, Castellarnau Font A, Barallat Gimeno E, Bosch Estrada T. Transdermal major opioid delivery in Primary Care. A review from 1997 to 2004. ABS Balaguer 13(3).

23. Dillie KS, Fleming MF, Mundt MP, French MT. Quality of life associated with daily opioid therapy in a primary care chronic pain sample. Journal of the American Board Family Medicine 2008;21:108-17.

24. Toquero de la Torre F, Zarco J. Atención Primaria de Calidad. Guía de práctica clínica en Dolor y su tratamiento. Madrid: IM\&C; 2004.

25. Montes E, Plasencia M, Moreno, Robaina F, Perez M, Aguiar JA, et. Al. Dolor crónico no oncológico. Boletín canario de uso racional del medicamento del servicio canario salud; 2010:2.

26. Chilet-Rosell E, Ruiz-Cantero MT, Fernández Sáez J, Álvarez-Dardet C. Inequality in analgesic prescription in Spain. A gender development issue. Gac Sanit 2013;27:135-142.

27. CADIME. Uso de opioides en dolor oncológico. Boletín Terapéutico Andaluz 2007;23:3.

28. Ruiz López D, Alonso Babarro A, Gómez Salcedo P, Varela Cerdeira M. Evolución del consumo de opiáceos en dos áreas sanitarias de Atención Primaria de la Comunidad de Madrid (España) y sus hospitales de referencia entre los años 2002 y 2006. Rev Clin Esp 2008;208:541-5.

29. Gómez Salcedo P, Herrero Ambrosio A, Muñoz Ramón JM. Estudio de utilización de analgésicos opiáceos en un hospital general universitario. Rev Soc Esp Dolor 2009;16(7):373-80.

30. De Conno F, Ripamonti C, Brunelli C. Opioid purchases and expenditure in nine western European countries: «Are we killing off morphine?» Palliat Med 2005;19:179-84.

31. British Pain Society. Opioids for persistent pain: Good practice; 2010. URL: http://www.britishpainsociety.org/ book_opioid_main.pdf [5/3/13] Revised 2013. 